\title{
NUMERICAL MODELLING OF SILICON MELT PURIFICATION IN INDUCTION DIRECTIONAL SOLIDIFICATION SYSTEM
}

\author{
G. Djambazov ${ }^{(1)}$, V. Bojarevics ${ }^{(1)}$, K. Pericleous ${ }^{(1)}$ and M. Forzan ${ }^{(2)}$ \\ ${ }^{(1)}$ University of Greenwich, Park Row, London, SE10 9LS, United Kingdom \\ ${ }^{(2)}$ Universita degli Studi di Padova, Via 8 Febbraio 1848, 2, Padova, 35122, Italy
}

\begin{abstract}
Solar grade silicon production is an energy intensive and harmful to the environment process. Yet $40 \%$ of this valuable product material is lost into sawdust (kerf loss) during wafering. The kerf waste from Fixed Abrasive Sawing of PV silicon wafers is pelletized and then remelted in an induction furnace. The furnace has a square cross-section quartz crucible, surrounded by graphite susceptors and heated by an induction coil that enables directional solidification of the new ingot. Top and bottom 'pancake' coils provide additional temperature control. Once melted, silicon becomes electrically conductive and subject to stirring by induction. To recycle the silicon, particulate impurities (due to the sawing, condensed silicon oxides or carbides) need to be removed. Flow control and the electromagnetic Leenov-Kolin force are used to expel particulates, through a novel dual frequency induction scheme. Three-dimensional, multi-physics numerical modelling captures the electromagnetic, fluid-flow and heat-transfer effects in this process. The presented results show it is possible to retain the impurity particles on the sides of the solidified ingot where they can be sliced off and removed.
\end{abstract}

\section{INTRODUCTION}

The process of solar grade silicon production from $\mathrm{SiO}_{2}$ is energy intensive (typically 50 $\mathrm{kWh} / \mathrm{kg}[1])$ and harmful to the environment. Yet, $40 \%-50 \%$ of this valuable product material is lost into sawdust (kerf loss) during wafering [2,3]. It is desirable to remelt the sawdust in a process which also removes impurities. Various laboratory methods, e.g. chemical treatment, heavy fluid centrifugation, electric field, phase-transfer, organic solvent separation, have been tried for removing the SiC particles from the kerf silicon [2]. The emerging technology, Fixed Abrasive Sawing, which uses diamond particles (instead of $\mathrm{SiC}$ ) fixed to a cutting wire to slice the silicon feedstock presents an opportunity for industrial-scale recycling [3]. The kerf loss from this process can be pelletized and then remelted and overheated in a vacuum induction furnace to remove organics and the silicon dioxide which inevitably forms during the sawing [4]. This leaves about $200 \mathrm{ppm}$ of diamond particles. If left untreated, formation of silicon carbide and precipitation during crystallisation is to be expected. The scheme proposed in the EU SIKELOR project [4] involves the careful control of induction currents which penetrate the melt to direct non-conducting particles towards the walls of the crucible using the electromagnetic Leenov-Kolin force [5] and so can aid the separation of the particles from the bulk of the melt $[6,7]$. 
The investigated design of an induction furnace (iDSS) aims to produce solar grade multicrystalline silicon blocks cast in commercial sizes. It has a square cross-section quartz crucible which is surrounded by graphite susceptors forming an air-tight and thermally-insulated block [8]. On the outside, the block is surrounded by six water-cooled induction coils which can be independently switched on and off (Figure 1). That enables directional solidification of the new ingot. Top and bottom 'pancake' coils provide additional temperature control. Once melted, silicon becomes electrically conductive and therefore subject to stirring by the electromagnetic Lorentz force. A novel dual frequency induction scheme is investigated. Low frequency and high frequency electrical currents are simultaneously applied to the coils. The low frequency current induces stirring of the silicon melt while the high frequency current heats the melt through the graphite susceptors. This is possible because the higher electrical resistance of the graphite allows the low-frequency magnetic field to penetrate the silicon charge with sufficient strength. The following sections present the mathematical model of the dual frequency induction and the computed results for a given scenario of the switching of the coils for directional solidification.

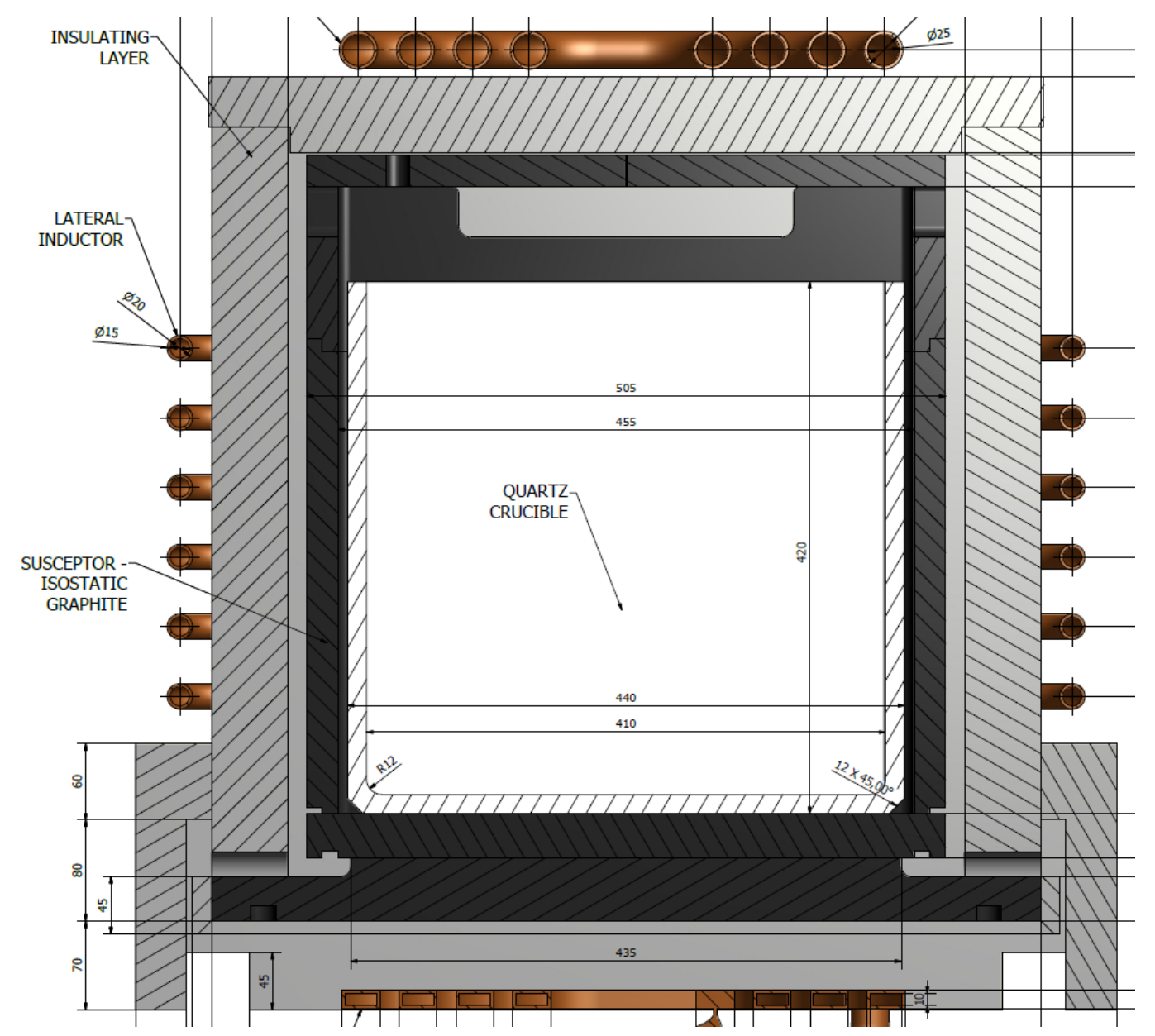

Figure 1. Cross-section of iDSS furnace with top, bottom and side coils

\section{MATHEMATICAL AND NUMERICAL MODEL}

\section{Electromagnetic field}

A new formulation [9] of the electromagnetic field equations is used which allows the computational domain to cover only the inside of the furnace and not the surrounding space. Pseudo-steady solutions are obtained for the unknown vector of the induced electric field $\boldsymbol{E}$ 
with its real (R) and imaginary (I) parts for a fixed angular frequency $\omega$ by solving six scalar equations:

$$
\nabla^{2}\left(\overrightarrow{E_{R, I}}-\nabla \varphi_{R, I}\right)= \pm \mu \sigma \omega\left(\overrightarrow{E_{I, R}}-\nabla \varphi_{I, R}\right)
$$

where $\mu$ and $\sigma$ are the magnetic permeability of the diamagnetic materials and their electrical conductivity. In the conducting parts of the domain, charge continuity is ensured by solving two auxiliary equations for the parts of a scalar electric potential: $\nabla^{2} \varphi_{R, I}=\nabla \cdot \overrightarrow{E_{R, I}}$. The induced magnetic field $\boldsymbol{B}$ is recovered from the solved electric field using Faraday's law:

$$
\omega \overrightarrow{B_{R, I}}=\mp \nabla \times \overrightarrow{E_{I, R}} \text {. }
$$

Boundary conditions on the external surfaces of the graphite susceptor box include the contributions to the local values of the magnetic field components by both the applied and the induced currents. Those contributions are calculated from the Biot-Savart integral [9]. Zero normal current conditions are imposed on the surfaces between conducting and non-conducting parts of the domain. The electrical current induced in the conducting liquid and solid objects is $\vec{J}=\sigma(\vec{E}-\nabla \varphi)$; the electromagnetically generated instantaneous Lorentz force $\boldsymbol{F}$ and Joule heat $Q$ are $\vec{F}=\vec{J} \times \vec{B}$ and $Q=J^{2} / \sigma$. Time-averaging these sinusoidal quantities yields the mean (pseudo-steady) values $\vec{F}_{\text {mean }}=0.5\left(\overrightarrow{J_{R}} \times \overrightarrow{B_{R}}+\overrightarrow{J_{I}} \times \overrightarrow{B_{I}}\right)$ and $Q_{\text {mean }}=\left(J_{R}^{2}+J_{I}^{2}\right) / 2 \sigma$. The mean force is responsible for the flow in the liquid and Joule heat is released both in the silicon and in the graphite susceptors which then transfer the heat by thermal radiation and conduction to the quartz crucible and the silicon charge.

\section{Fluid Flow and Turbulence}

Mass continuity in the liquid is achieved by ensuring the velocity $\boldsymbol{u}$ satisfies $\nabla \cdot \vec{u}=0$ which is an implicit equation for the pressure $p$ also appearing in the momentum equations [10]. The driving Lorentz force appears as a source term in the momentum equations. The effective viscosity which is responsible for the resistance to the flow includes the mixing action of turbulence (in spreading momentum between faster and slower fluid regions). The standard in CFD $k-\varepsilon$ model of turbulence is used with logarithmic-law wall functions on solid surfaces bounding the liquid. The numerical method used to solve the electromagnetic, fluidflow and turbulence model equations is based on finite-volume cell-centered discretization and velocity-pressure coupling is accomplished with the SIMPLE procedure and the RhieChow interpolation scheme [10].

\section{Forces on Particles}

As a first approximation, only the two main forces acting on the particles are considered namely the Leenov-Kolin force and fluid drag. The Leenov-Kolin force is a direct consequence of the Lorentz force $\boldsymbol{F}$ acting on the conducting liquid surrounding the non-conducting particle. It is caused by the pressure gradient across the particle as current is diverted around it and is oppositely directed to the Lorentz force vector: $\overrightarrow{F_{E}}=-V_{P} \frac{3}{2} \vec{F}_{\text {mean }}, V_{p}$ being the volume of the particle. This formula is valid for alternating currents and magnetic fields [11]; the original Leenov-Kolin expression [5] derived for direct current is two times weaker and has a coefficient $\frac{3}{4}$. The fluid drag force is expressed as $\overrightarrow{F_{D}}=C_{D} \rho_{L} \frac{A}{2}\left(\vec{u}-\overrightarrow{u_{P}}\right)\left|\vec{u}-\overrightarrow{u_{P}}\right|$ where $A$ is the crosssection area of the particle, $\boldsymbol{u}_{p}$ is the particle velocity and $\rho_{L}$ is the density of the liquid. The drag coefficient is $C_{D}=\frac{24}{R e}\left(1+0.15 R e^{0.687}\right)$ [12]. The maximum drag occurs when the particle is held stationary by the combination of Leenov-Kolin and adhesion forces, i.e. when $\overrightarrow{u_{P}}=0$. Since the aim is to predict whether the Leenov-Kolin force will be sufficiently strong to counteract the drag, the adhesion forces are not included in the force balance calculation. 


\section{Computational Domain}

After the initial melting of the silicon pellets, the liquid level reaches about $230 \mathrm{~mm}$ from the bottom of the crucible and its upper part remains empty. So we assume the top coil is no longer needed and is switched off. The electrical current in the bottom coil (Figure 1) is also switched off but its water flow remains on, thus allowing the bottom to become a heat sink promoting the directional solidification from the bottom up. The remaining six side coils can be switched off one by one from the bottom upwards as the solidification front progresses.

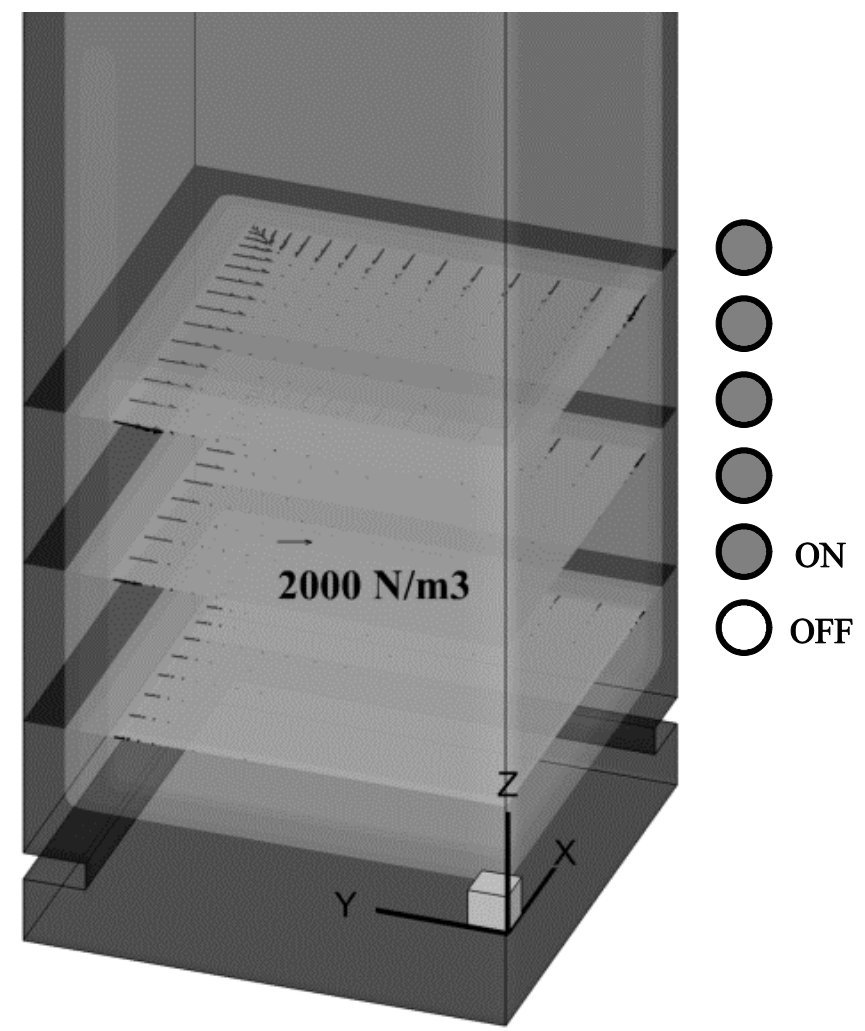

Figure 2. Outline of computational domain with side coils (on/off) and typical magnitude and distribution of Lorentz force in 3 horizontal sections through the liquid zone.

Preliminary simulations have shown that the effect of the coil leads can be ignored because their magnetic field cancels out (the same currents flowing in opposite directions) and the six turns can be represented in the model as exactly symmetrical. Since the furnace geometry is also symmetrical, it is enough to model only one quarter (Figure 2) which allows much shorter computational times. At the same time, for the calculation of the magnetic field, the currents in the whole furnace and of the whole coil are taken into account in the Biot-Savart integrals.

\section{RESULTS AND DISCUSSION}

Simulations were carried out for four different positions of the solidification front in order to reveal how the forces on the particles change during the directional solidification of the ingot: initial state (all liquid), 20, 40 and $60 \mathrm{~mm}$ from the bottom.

The step when only the lowest of the six side coils is switched off was chosen (Figure 2) in order to investigate the conditions for capturing the contaminating particles at the sides of the ingot early on in the solidification process. 
A frequency combination was examined for each of the front positions where both a high frequency $(2350 \mathrm{~Hz}, 1273 \mathrm{~A}$ amplitude) and a low frequency $(120 \mathrm{~Hz}, 500 \mathrm{~A})$ are provided by a special power supply. This is done with the following facts in mind: The magnetic field induced by the high frequency has shallow penetration and produces a lot of heat mainly in the graphite susceptors but is also responsible for the strong Lorentz (hence, Leenov-Kolin) force in a thin boundary layer in the silicon at the crucible walls, while the magnetic field of the lowfrequency current penetrates deep into the melt and is responsible for its stirring.

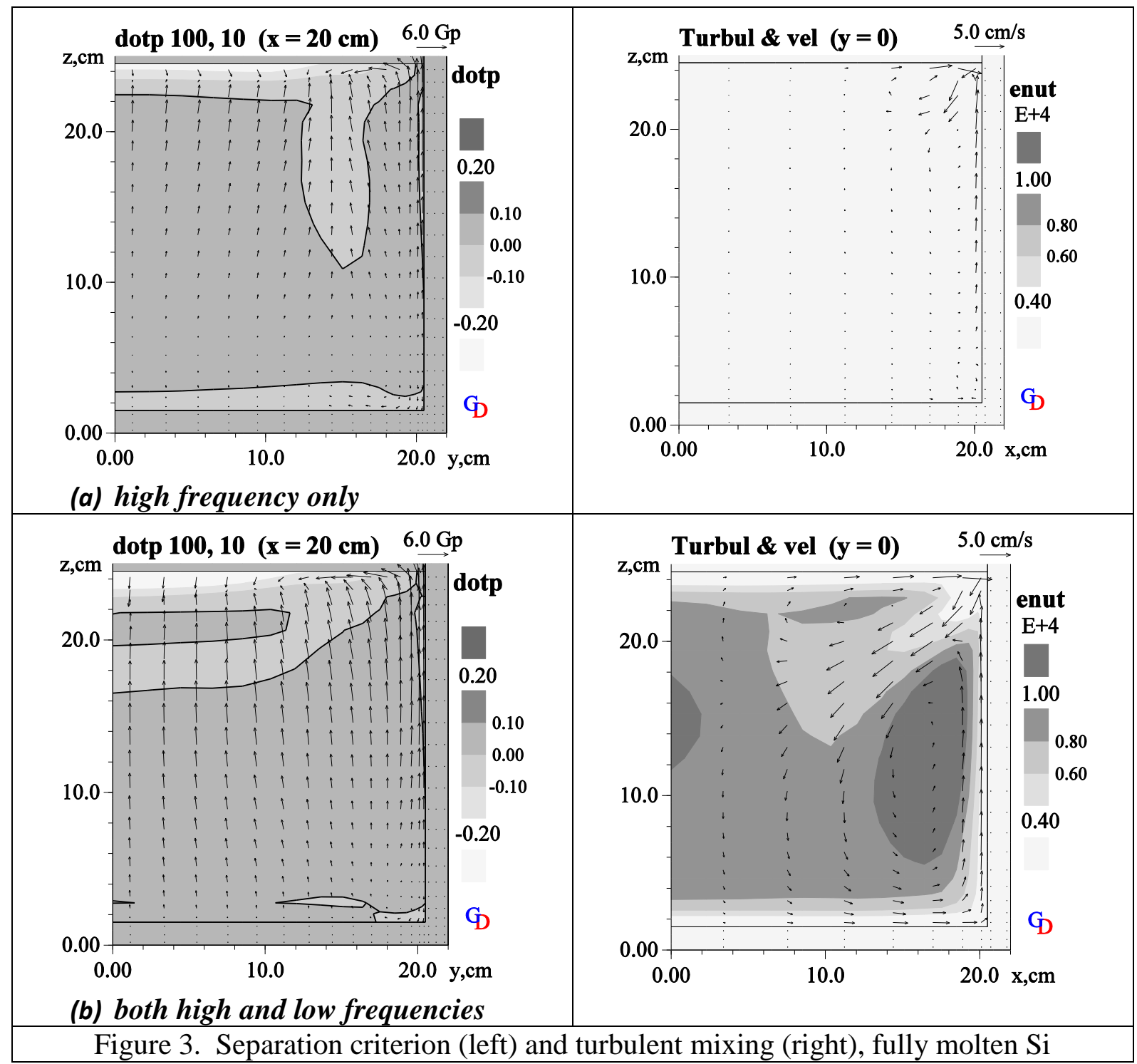

Two important aspects of the purification process occur simultaneously during the directional solidification of the silicon ingot: stirring of the melt, so that particulate impurities are taken from the bulk to the walls of the holding vessel (crucible) and entrapment of the particles by the Leenov-Kolin force at the walls. (These contaminated sides of the solidified ingot can be sliced off, leaving the purified bulk.) Good stirring is achieved with higher liquid velocities (mainly driven by the Lorentz force) and more vigorous turbulent mixing indicated by the 'effective turbulent viscosity' (enut) calculated via the turbulence model. A special 'separation criterion' was defined to indicate the strength of the particle entrapment - the dot product of the Leenov-Kolin force and the fluid drag force: 


$$
\operatorname{dotp}=\frac{\overrightarrow{F_{E}} \cdot \overrightarrow{F_{D}}}{s^{2}}
$$

where $s$ is a scaling factor which is chosen as the weight of the considered particle $\left(\mathrm{G}_{\mathrm{p}}\right)$. Where the dot product is positive, the flow helps push the particles towards the entrapment zones; in regions of negative dot product, the flow takes the particles back to the bulk of the melt.

Particle sizes of $100 \mu \mathrm{m}$ (shaded contours) and $10 \mu \mathrm{m}$ (line contours at value 0 ) are considered for comparison (on the left) in Figures 3 and 4. For the separation criterion 'dotp' the displayed cross-sections are $4 \mathrm{~mm}$ from the crucible wall $(\mathrm{x}=200 \mathrm{~mm})$. On the right, the corresponding turbulent mixing contours and liquid velocity vectors are shown for cross-sections through the middle of the domain $(y=0)$.

In Figure 3 a comparison is shown between the conditions achieved with a single high frequency (top part $a$ ) and with a combination of high and low frequencies applied simultaneously (bottom part $b$ ). The conditions relate to mixing (indicated by the velocity field and turbulence effective viscosity, seen on the right) and particle separation (indicated by the resultant forces on the particles and the values of the separation criterion, seen on the left).

It can be seen on Figure 3 that the addition of the low frequency enhances the mixing substantially which is good for taking the non-conducting particles out of the bulk. At the same time, the dual frequency electromagnetic field decreased the area of negative dot-product which means the retention of particles was also improved.

Figure 4 shows that the good separation when both high and low frequencies are applied is sustained during the growth of the silicon ingot - a continuous zone of positive 'dotp' persists just above the advancing solid front which means particles coming out of the liquid bulk will be trapped by the Leenov-Kolin force at the crucible wall as planned. The velocity fields and the amount of turbulence seen on the right of Figure 4 indicate sufficient mixing, so all particles can be transported from the bulk of the melt to the retaining walls.

\section{CONCLUSIONS}

The problem of impurity particles expulsion from recycled kerf loss of PV silicon is addressed by computational numerical modelling based on solving partial differential equations for the electric field and liquid velocity vectors in induction furnaces with one or two imposed frequencies. The model predicts that a combination of a high frequency (e.g. $2.3 \mathrm{kHz}$ ) current and a low frequency (e.g. $120 \mathrm{~Hz}$ ) current applied simultaneously to the side inductor coils of the directional solidification furnace can achieve good retention of the particles at the walls of the holding vessel while simultaneously stirring the bulk of the melt so that all particles have significant chances of reaching the walls. This allows, after solidification, the sides of the new ingot to be removed by slicing making the purified silicon core available for reuse.

The use of dual frequencies (low and high) provides a measure of independent control between heating, stirring and separation. Further work is in progress to optimise the process.

\section{ACKNOWLEDGEMENTS}

This work is financed by SIKELOR [4] - a project funded by the 7th Framework Programme of the European Commission, sub-programme: ENV.2013.6.3-1, project reference 603718. 


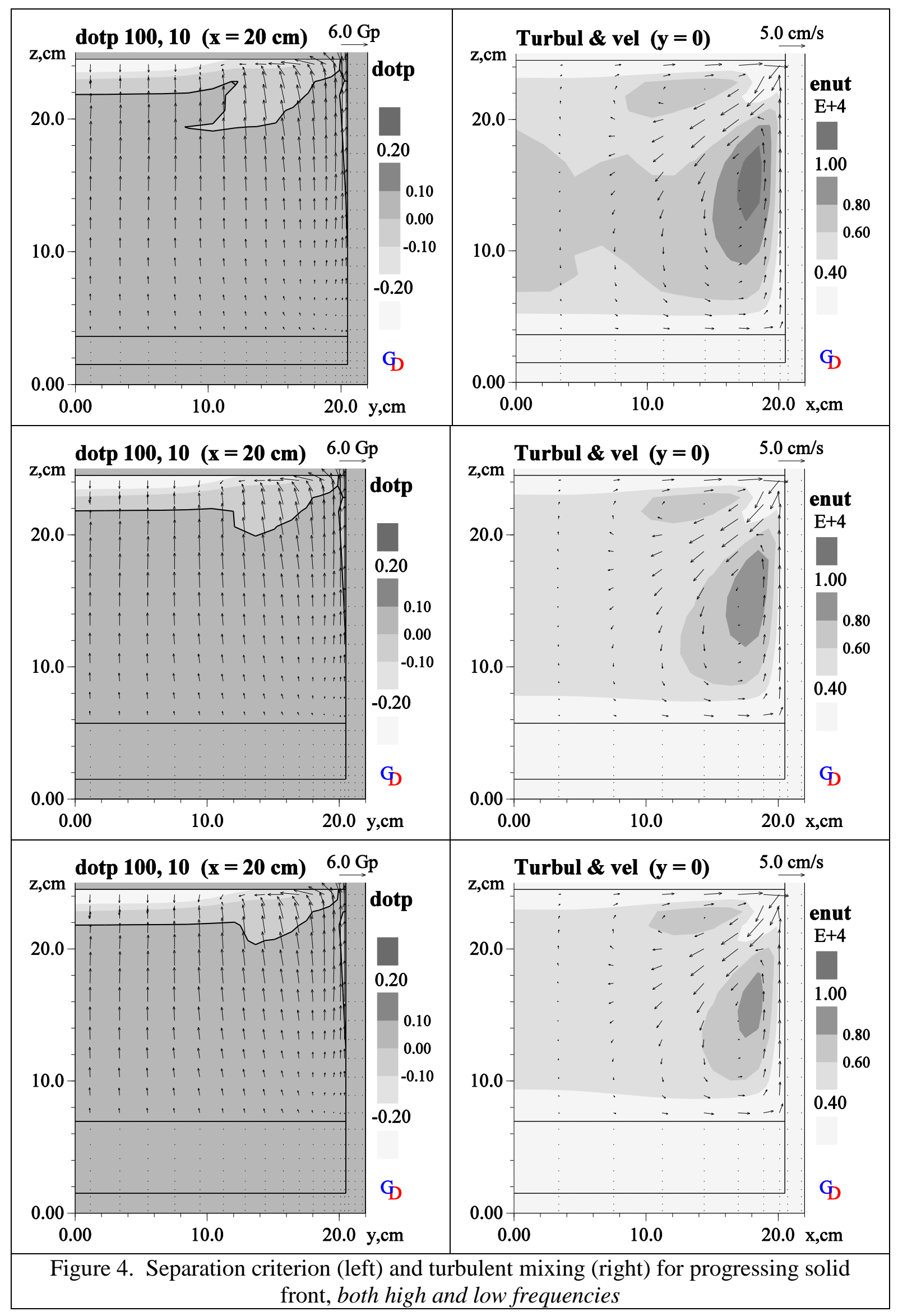




\section{REFERENCES}

[1] Odden, J.O., Halvorsen, G., Rong, H., Gløckner, R. (2008). Comparison of the energy consumption in different production processes for solar grade silicon. Silicon for the Chemical and Solar Industry IX conf. Oslo, Norway.

[2] Xing, P.-F., Guo, J., Zhuang, Y.-X., Li, F., Tu, G-F. (2013). Rapid recovery of polycrystalline silicon from kerf loss slurry using double-layer organic solvent sedimentation method," International Journal of Minerals, Metallurgy and Materials, 20, (10), 947-952.

[3] Lombardi, I., Fragiacomo, G., Zehetmeier, C., Bye, J.-I., Nielsen, Ø., Rohr, C., Gäumann, B., Künzli, A. (2009). High yield recycling process of silicon kerf from diamond wire wafering. Proceedings of the 24th European Photovoltaic Solar Energy Conference, Hamburg, Germany, 21-25 September 2009, 1256 -1258.

[4] SIKELOR. Silicon kerf loss recycling. www.sikelor.eu

[5] Leenov, D., Kolin, A. Theory of electromagnetophoresis. (1954). Journ. Chem. Phys., 22 (4), 683-688.

[6] Moffatt, H.K., Sellier, A. (2002). Migration of an insulating particle under the action of uniform ambient electric and magnetic fields. Part 1. General theory. J. Fluid Mech., 464, 279-286.

[7] Taniguchi, S., Yoshikawa, N., Takahashi, K. (2005) . Application of EPM to the separation of inclusion particles from liquid metal. The 15th Riga and 6th PAMIR

Conference on Fundamental and Applied MHD, Alemany, A., Ed., Salaspils Institute of Physics, Riga, Latvia, p. 55. http://pamir.sal.lv/2005/cd/vol.I/riga-pamir-vol.I-55.pdf

[8] Dughiero, F., Forzan, M, Pozza, C., Tolomio, A. (2014). Experimental results in industrial environment of the i-DSS furnace. Conference: Modelling for Electromagnetic Processing, Hannover, Germany, 2014. http://www.sikelor.eu/publications

[9] Djambazov, G., Bojarevics, V., Pericleous, K., Croft, T.N. (2015). Finite volume solutions for electromagnetic induction processing. Applied Mathematical Modelling, 39, 4733-4745.

[10] PHYSICA Theory Manual. http://staffweb.cms.gre.ac.uk/ physica/phy3.11/theory/

[11] Bojarevics, V., Pericleous, K. (2015). Particle tracking during melting and solidification in the presence of AC magnetic field of various configurations. Proceedings 8th Int. Conf. Electromagnetic Processing of Materials, 2015, Cannes, France.

[12] Cross, M., Croft, T.N., Djambazov, G., Pericleous, K. (2006). Computational modelling of bubbles, droplets and particles in metals reduction and refining. Applied Math. Modelling, 30, 1445-1458. 